\title{
Predictors for Recurrence of Chronic Subdural Hematoma
}

\author{
Alexander HAMMER ${ }^{1}$, Alexander TREGUBOW ${ }^{1}$, Ghassan KERRY ${ }^{1}$, Michael SCHREY ${ }^{1}$, Christian HAMMER ${ }^{2}$, \\ Hans-Herbert STEINER \\ ${ }^{1}$ Paracelsus Medical University, Department of Neurosurgery, Nürnberg, Germany \\ ${ }^{2}$ University of Erlangen-Nürnberg, Department of Anatomy 2, Erlangen, Germany
}

Parts of this study have been presented as a scientific poster at "Paracelsus Science Get Together 2014" in Salzburg, Austria.

\section{ABSTRACT}

AIM: This prospective study was designed to analyze the dependence of different factors on the recurrence rate of chronic subdural hematoma $(\mathrm{cSDH})$ after surgical treatment.

MATERIAL and METHODS: Seventy-three consecutive patients, who were surgically treated at our department due to cSDH between 2009 and 2012, were included. The following parameters were analyzed: patient age and gender, occurrence of trauma, time between trauma and admission, neurological symptoms, presence of minor diseases, intake of anticoagulation medication. We classified the results of diagnostic imaging and determined the space-consuming effect via the cerebral midline shift. In addition, we scrutinized intraoperative findings and the dependence of the position of subdural drainage on the recurrence rate of cSDH.

RESULTS: In our patient group, cSDH recurrence was significantly associated with aphasia $(\mathrm{p}=0.008)$. Moreover an increased cSDH recurrence rate was observed in the patient group that had a separated manifestation of the cSDH in the preoperative diagnostic imaging $(p=0.048)$ and received no drainage implant $(p=0.016)$. Homogeneous isodense cSDH was associated with no apparent recurrence $(p=0.037)$.

CONCLUSION: Within the scope of this study, we detected aphasia and separated cSDH as predictors of cSDH recurrence. Homogeneous isodense cSDH seems to be a good prognostic sign regarding the risk of recurrence development. Furthermore, our data clearly emphasize the importance of surgically applied drainage implants to prevent a recurrence of cSDH.

KEYWORDS: Chronic subdural hematoma, Diagnostic imaging, Subdural drainage, Recurrence, Aphasia

\section{INTRODUCTION}

$\longrightarrow$ hronic subdural hematoma $(\mathrm{cSDH})$ is one of the most common forms of intracranial hemorrhage. It predominantly occurs at an older age and is still associated with a significant morbidity. Currently, the incidence of surgically treated cases worldwide is 5.3 per 100,000 inhabitants per year (14). The average age of cSDH manifestation lies between 56 and 63 years (23), but patients older than 70 years are affected most often $(8,20,30)$. Thus, the incidence of cSDH is positively correlated with patient age, with the highest rate being found in the $8^{\text {th }}$ decade of life (7.35 per 100,000 per year)(4). Overall, males are affected approximately twice as often as females $(2,25)$. In the $8^{\text {th }}$ decade of life, however, a male:female ratio of about $4: 1$ is observed.

Pathologically, a cSDH represents an accumulation of blood between the dura mater and the arachnoid mater. It displays different conditions of liquefaction and is usually surrounded by a more or less pronounced fibrin capsule, which forms approximately 2-3 weeks after primary intracranial injury associated with an acute subdural hematoma $(7,12)$. Consequently, a cSDH usually does not occur before 2-3 
weeks after trauma. However, in some cases posttraumatic symptoms take months to manifest. Sometimes, the patient does not remember a trauma at all. Then a cSDH is defined by criteria of diagnostic imaging. Despite numerous studies in the past $20-25$ years, there has been no significant change in the treatment and outcome of $\mathrm{CSDH}$. Moreover, the pathogenesis of cSDH recurrence and, hence, the best choice of medical intervention are still controversial. There have been several attempts to figure out how different factors may affect the genesis of cSDH $(1,8,9,15-20,25,28,30)$. In the corresponding studies, the dependence of the rate of cSDH recurrence on patient age, preoperative general and neurologic condition, co-morbidities, medication, choice of operative intervention and diagnostic imaging was evaluated. However, efficient assessment of the influence of different factors on $\mathrm{CSDH}$ recurrence has been limited because most studies were performed retrospectively.

The present prospective research study contributes to the identification of pivotal factors that may critically influence the cSDH recurrence rate.

\section{MATERIAL and METHODS}

We prospectively analyzed 73 consecutive patients of cSDH from January 2009 through December 2012 (approved via cranial computed tomography (CCT) scan), who underwent surgical treatment at our department. We did not exclude any case of operatively treated mono-lateral cSDH among that period.

We also analyzed the distribution of the patients with respect to the intake of anticoagulants, trauma anamnesis, neurological symptoms and regarding the presence of minor diseases such as arterial hypertension or cardiac arrhythmias besides cSDH.

Furthermore, we investigated the incidence of the different intraoperative morphological subtypes (oleaginous contents, bloody liquid contents, bloody contents with blood clots, watery xanthochromic contents).

In order to assess the CCT findings, we used the classification of Nakaguchi et al. (18). In addition, the space-consuming effect of the SDH was assessed based on the cerebral midline shift (MLS). This was measured at the level of the Foramina of Monro on the CCT images. Regarding the issue of investigating the space consuming effect, patients with double-sided SDH were excluded.

After the evacuation of the hematoma, a subdural drainage was implanted in 71 cases. In two patients, no drainage had been implanted; whereby one of them had pulled the drainage out immediately after surgery and was therefore counted as patient without drainage.

All patients were subjected to postoperative CCT control imaging. The interval between the CCT controls differed and depended on the CCT findings and the clinical condition of the patient. The CCT controls were usually continued until the hematoma had disappeared completely. In some cases, no further controls were performed if the subdural hematoma had no space consuming effect, caused no complaints, and the patients had been in a normal neurological condition for at least 6 weeks. The average post-operative follow-up lasted 57.8 days.

Cases were defined as relapses if a second operation had been necessary (e.g. due to an increase of the size of the residual hematoma and/or because of persisting neurological symptoms in association with a still existing, space-consuming hematoma residual). Acute postoperative hemorrhage has not been taken into account. Statistical analysis was performed with the $X^{2}$ - independence - test and Student's t-test. For that purpose, the "Microsoft Excel" software was used. Results have been regarded as significant if the $H_{0}$ hypothesis had a probability $\mathrm{p} \leq 0.05$.

The local medical association confirmed that this study is in accordance with the ethical standards laid down in the 1964 declaration of Helsinki and its later amendments.

\section{- RESULTS}

The patient collective consisted of 47 men and 26 women (ratio 1.8:1) at an average age of 69.1 (45-92) years and 75.7 (34-88) years, respectively. Overall, the average age of all patients examined was $71.5(34-92)$ years at the time of surgery. Most of the female patients were 81-90 years of age (14 patients), while the majority of male patients were aged 61-70 years (18 patients).

Among the 73 patients examined, hematoma recurrence was observed in 19 patients (26\%). Six out of a total of 26 women (23\%) and 13 out of a total of 47 men (28\%) exhibited SDH recurrence. The average age of the affected women was 77.5 years at the time of the recurrence, whereas the respective average age of male patients was 74.1 years. Among the female patients, recurrence occurred in the $7^{\text {th }}$ decade of life in 1 out of $3(33 \%)$, in the $8^{\text {th }}$ decade in 2 out of $7(29 \%)$ and in the $9^{\text {th }}$ decade in 3 out of $14(21 \%)$ cases. In the male group recurrence was diagnosed between 51 and 60 years of age in 1 out of $5(20 \%)$ patients, between 61 and 70 years in 4 out of $18(22 \%)$, between 71 and 80 years in 4 out of $9(44 \%)$ and between 81 and 90 years in 4 out of 8 patients (50\%). Nevertheless, statistical analysis did not show a significant dependency of the appearance of recurrence on an age greater than 70 years. $\left(H_{0}\right.$ : recurrence is independent of incidence of age > 70 years (male); $\mathrm{p}=0.068 ; \mathrm{X}^{2}$ - independence - test).

Table I illustrates the neurological symptoms at the time of the first diagnosis and shows the rate of the different symptoms in the patient groups with and without CSDH recurrence. Headache, disturbance of consciousness, vertigo, gait disorder and no symptoms had been represented more frequently in the group without recurrence when compared to the group of all patients. As opposed to this, paresis, aphasia, confusion and epilepsy were more often associated with a recurrence in contrast to the entire patient collective. The appearance of aphasia showed a highly significant association with $\mathrm{CSDH}$ recurrences $\left(\mathrm{H}_{0}\right.$ : recurrence is independent of incidence of disorders of speech; $p=0.008 ; X^{2}-$ independence - test). 
In our patient population, we found a homogeneous $\mathrm{cSDH}$ in 47 cases $(64 \%)$. Thirty-eight $(52 \%)$ of them were isodense, five $(7 \%)$ hyperdense and four (5\%) hypodense. In 14 cases $(19 \%)$ the inhomogeneous hematoma showed the trabecular subtype, in 5 cases $(7 \%)$ the laminar and in 7 cases $(10 \%)$ the separated subtype (Figure 1A-F).
The recurrence rate dependent on the initial CCT findings was $16 \%$ for the homogeneous isodense hematomas, $0 \%$ for the homogeneous hyperdense hematomas, $50 \%$ for the homogeneous hypodense hematomas, 36\% for the trabecular hematomas, $40 \%$ for the laminar hematomas, and $57 \%$ for the separated $\mathrm{cSDH}$ (Figure 2). Significance was obtained

Table I: Clinical Symptoms in the Patient Groups with and without cSDH Recurrence

\begin{tabular}{lccc}
\hline Symptoms & No-recurrence $(\mathbf{n}=\mathbf{5 4})$ & Recurrence $(\mathbf{n = 1 9 )}$ & Total $(\mathbf{n}=\mathbf{7 3})$ \\
\hline Headache & $22(40.7 \%)$ & $4(21.1 \%)$ & $26(35.6 \%)$ \\
\hline Paresis & $17(34.5 \%)$ & $9(47.4 \%)$ & $26(35.6 \%)$ \\
\hline Aphasia & & $6(31.6 \%)$ & $10(13.7 \%)$ \\
\hline Confusion $^{+}$ & $4(7.4 \%)$ & $5(26.3 \%)$ & $11(15.1 \%)$ \\
\hline Disturbance of consciousness & $6(11.1 \%)$ & $4(21.1 \%)$ & $19(26.0 \%)$ \\
\hline Epilepsy & $15(27.8 \%)$ & $2(10.5 \%)$ & $4(5.5 \%)$ \\
\hline Vertigo & $2(3.7 \%)$ & $1(5.3 \%)$ & $6(8.2 \%)$ \\
\hline Gait disorder & $5(9.3 \%)$ & $1(5.3 \%)$ & $13(17.8 \%)$ \\
\hline No symptoms & $12(22.2 \%)$ & $1(5.3 \%)$ & $6(8.2 \%)$ \\
\hline
\end{tabular}

Aphasia $(\dagger)$ was associated with the appearance of recurrence $\left(\boldsymbol{H}_{0}:\right.$ Recurrence is independent of incidence of aphasia; $p=0.008 ; X^{2}-$ independence - test).
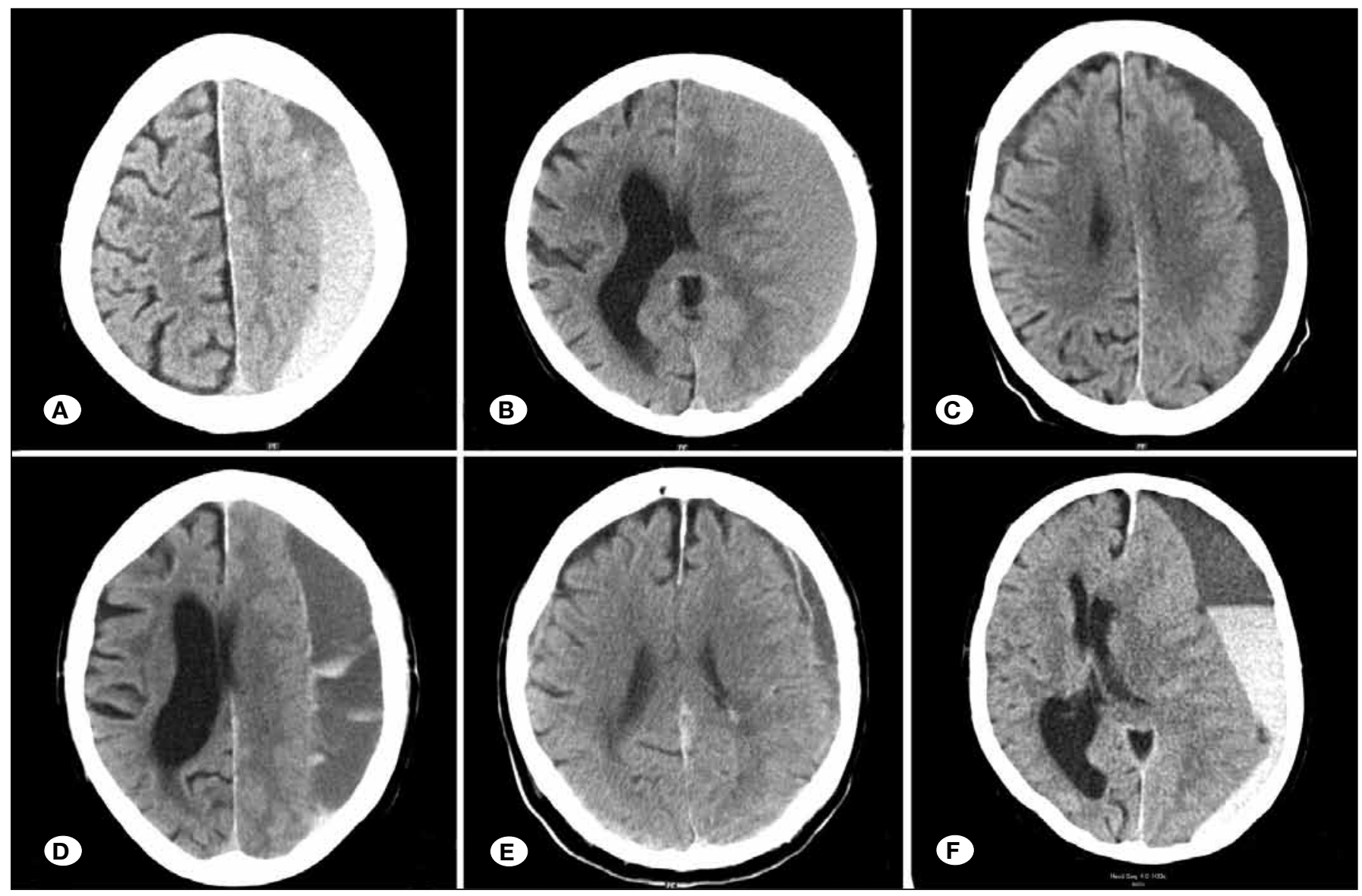

Figure 1: Computed tomography scan of the skull showing the different types of cSDH. A) homogeneous hyperdense, B) homogeneous isodense, C) homogeneous hypodense, D) trabecular, E) laminar, F) separated cSDH. 
regarding an association of homogeneous isodense $\mathrm{CSDH}$ with the appearance of no recurrence $\left(H_{0}\right.$ : no recurrence is independent of homogeneous isodense cSDH; $p=0.038 ; X^{2}-$ independence - test), as well as the association of separated cSDH and the appearance of recurrence $\left(H_{0}\right.$ : recurrence is independent of incidence of separated cSDH; $p=0.048 ; X^{2}-$ independence - test). All relapsed cSDHs had a more or less pronounced fibrin capsule.

Eleven recurrences have been on the left side, four on the right side. From the initial four cSDH cases on both sides which recurred, two recurred on both sides and two on the left side.

In 73 patients 94 surgeries had been performed. Nineteen patients had been operated for the second time because of $\mathrm{SDH}$ recurrence and one patient due to a subdural empyema. One patient underwent surgery for the third time because of a subdural empyema. During a total of 92 operations on cSDH a burr hole had been drilled in 82 cases $(89 \%)$, while in 10 cases $(11 \%)$ an osteoplastic trepanation had been performed. The first surgery was constituted by burr hole trepanation in 67 cases $(92 \%)$, and by osteoplastic trepanation in the remaining 6 cases (8\%). During the second operation SDH drainage was administered via the old hole in 15 cases $(79 \%)$ and via a trepanation in 4 cases (21\%). Regarding the first operation, no drainage was implanted in two cases due to the rapid expansion of the brain to the normal level in on case; in the other case, the patient pulled the drainage out immediately after surgery and was therefore counted as patient without drainage. An increased cSDH recurrence rate occurred if no drainage had been implanted $\left(\mathrm{H}_{0}\right.$ : recurrence is independent of incidence of no drain; $p=0.016 ; X^{2}-$ independence - test).

There was no significant connection detectable regarding the drainage positions, appearance of trauma, minor diseases, intake of coagulation-impeding medication, average midline shift and hematoma content $\left(X^{2}-\right.$ independence - tests; Student's t-test for "midline shift - recurrence").

Table II shows the clinical, radiological and pathological findings of the cases of recurrence.

\section{DISCUSSION}

In the literature, the recurrence rate varies between $39 \%$ and $8 \%$ (average 16\%); most authors report values around $10 \%$ $(1,8-10,17,24,26)$. In our collective of 73 patients, there was a relapse of cSDH in 19 cases (26\%).

Theoretically, the pronounced age-related brain atrophy could lead to a slow and insufficient expansion of the brain after surgery and, therefore, to a slow increase of intracranial pressure, which might lead to a higher risk of SDH recurrence development. In our study, the recurrence of CSDH appeared in male patients in the ninth decade in $50 \%$ of the cases. Nevertheless, we did not detect a significant association with the appearance of recurrence for male patients $>70$ years $\left(p=0.068 ; X^{2}-\right.$ independence - test).

All patients (with one exception) who developed a cSDH relapse had neurological symptoms of various sorts and combinations throughout their hospital stay. The most common neurological symptom correlating with recurrences was paresis (Table I), which was exhibited by $47.4 \%$ of the relapsed patients (total $35.6 \%)$. In the group of patients with recurrence, aphasia had been observed more frequently $(31.6 \%)$ as compared to the whole patient collective (13.7\%). Moreover, $60 \%$ of the cSDH patients with aphasia developed $\mathrm{CSDH}$ recurrence. These numbers suggest that palsy and aphasia may constitute warning signs for CSDH recurrence. Only the dependence of

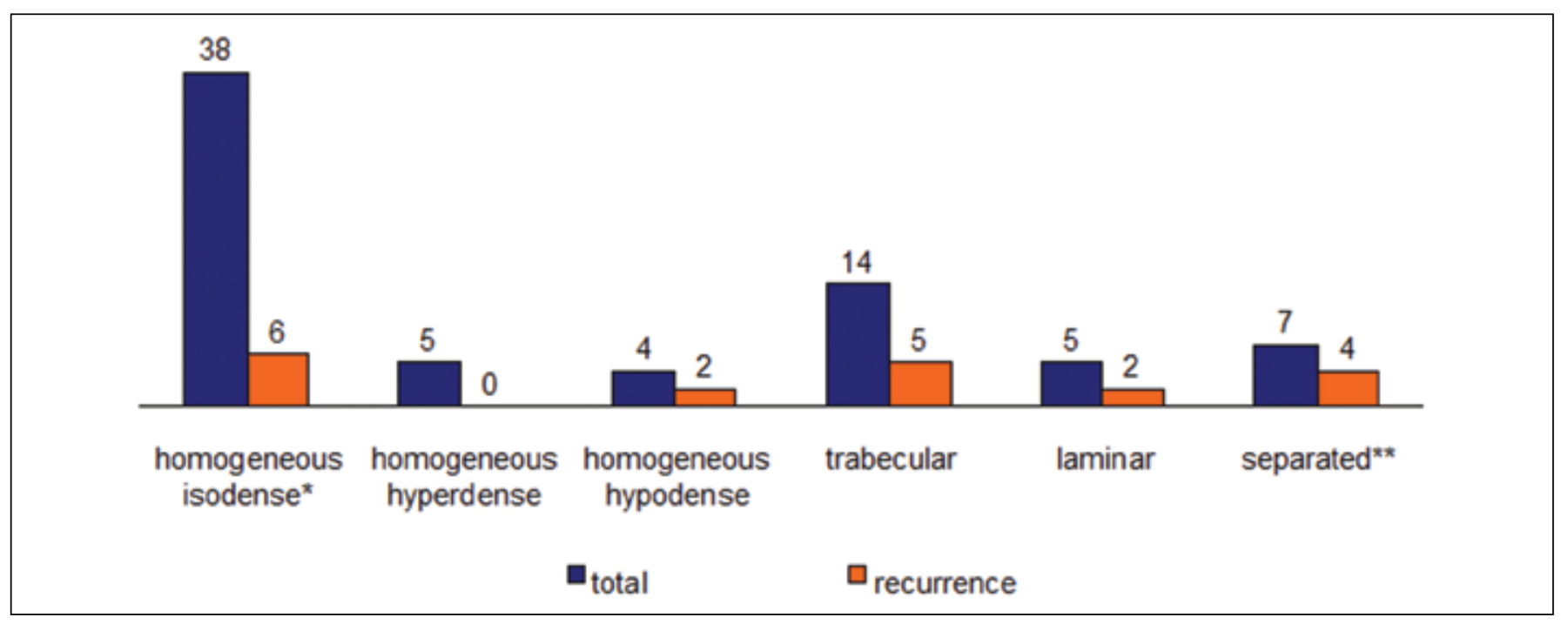

Figure 2: Recurrence rate dependent on the findings in CCT (homogeneous isodense, homogeneous hyperdense, homogeneous hypodense, trabecular, laminar, separated). Homogeneous isodense $\mathrm{CSDH}\left({ }^{*}\right)$ was associated with no appearance of recurrence $\left(\mathrm{H}_{0}\right.$ : no recurrence is independent of incidence of homogeneous isodense $c S D H ; p=0.038 ; X^{2}-$ independence - test). Separated cSDH $\left.{ }^{\star \star}\right)$ was associated with the appearance of recurrence $\left(\mathrm{H}_{0}\right.$ : recurrence is independent of incidence of separated cSDH; $\mathrm{p}=0.048 ; \mathrm{X}^{2}-$ independence - test). 
aphasia on the appearance of recurrence was highly significant $\left(p=0.008 ; X^{2}\right.$ - independence - test). In our patient collective, all patients with aphasia also exhibited a left-sided chronic subdural hematoma. Most hematomas showed a significant frontobasal expansion. Stanisic et al. (25) and Nakaguchi et al. (18) detected an association between basal chronic subdural hematoma and recurrence. In this context, the authors stated that patients with basal chronic subdural hematoma are suffering from a higher re-bleeding rate because of a greater extent of feeding vessels from the middle meningeal artery and because of possible bleedings due to traumatized membrane at the time of catheter implantation $(18,25)$. Moreover, a greater technical difficulty in evacuation of these hematomas with possible insufficient evacuation might be a cause for the higher recurrence rate $(18,25)$. Taken together, this may also partially explain the correlation of speech disorders with high recurrence rates.

Regarding the neurological symptom palsy, other authors did not acknowledge motor failures to be crucial in connection with cSDH recurrence, either. Oishi et al. point out that $60 \%$ of all cSDH patients, irrespective of recurrence development, show an initial paresis. They furthermore reported initial headaches to constitute the most prominent distinguishing feature that $50 \%$ of the recurrence patients suffered from, as compared to only $26 \%$ in the recurrence-free cohort (20). However, in other studies, a clear connection between initial headache and a low recurrence rate of cSDH was revealed $(3,16,21)$. In our patient population $41 \%$ of the recurrencefree patients complained about initial headache, but only $21 \%$ of the relapsed patients (total: $35.6 \%$ ). However, statistical analysis revealed no significant connection of headache and appearance of recurrence in our study.

A correlation of the $\mathrm{CSDH}$ recurrence rate with the preoperative CCT features of the hematoma has often been described in literature. Oishi et al. report that in an examined collective of 116 patients $17 \%$ of the operated hyperdense cSDH relapsed. There was no relapse among the hypodense hematomas (20). Ko et al. divided their cSDH cases into 2 groups. The first group comprised the mixed and hyperdense hematomas, while the second group was composed of isodense and hypodense hematoma. The recurrence rate was $23.5 \%$ in the first group and only $2.4 \%$ in the second (9). In addition, Stanisic et al. acknowledge that CCT is important for the prediction of $\mathrm{SDH}$ recurrence. They report that a relapse developed more frequently in their patient collective with separated cSDH. A basal blood clot, as visualized by postoperative CCT, is also commonly considered as a bad prognostic sign in terms of $\mathrm{SDH}$ relapse development (25). The group of Nakaguchi

Table II: Clinical, Radiological and Pathological Characteristic of the Cases of Recurrence

\begin{tabular}{|c|c|c|c|c|c|}
\hline Patient No & Clinical deficit & Paresis & Aphasia & Radiological findings & Pathological findings \\
\hline 2 & Yes & No & No & separated & oleaginous contents \\
\hline 3 & Yes & Yes & Yes & trabecular & oleaginous contents \\
\hline 4 & Yes & Yes & No & separated & oleaginous contents \\
\hline 6 & Yes & No & No & trabecular & oleaginous contents \\
\hline 7 & Yes & Yes & No & trabecular & watery xanthochromic contents \\
\hline 8 & Yes & Yes & No & homogeneous isodense & oleaginous contents \\
\hline 9 & Yes & Yes & Yes & laminar & bloody liquid contents \\
\hline 12 & No & No & No & homogeneous isodense & oleaginous contents \\
\hline 13 & Yes & Yes & Yes & trabecular & bloody liquid contents \\
\hline 14 & Yes & No & No & homogeneous hypodense & oleaginous contents \\
\hline 15 & Yes & No & No & separated & oleaginous contents \\
\hline 16 & Yes & Yes & Yes & laminar & oleaginous contents \\
\hline 17 & Yes & No & No & homogeneous isodense & oleaginous contents \\
\hline 18 & Yes & No & No & trabecular & oleaginous contents \\
\hline 19 & Yes & No & No & homogeneous isodense & watery xanthochromic contents \\
\hline
\end{tabular}


employed CCT data to divide the CSDH into homogeneous, laminar, separated and trabecular CSDH. The separated SDH showed the highest and the trabecular the lowest recurrence rate (18). In our patient population, we observed a significant dependency of the recurrence rate on the separated cSDHs $\left(p=0.048 ; X^{2}-\right.$ independence - test) as well as a significance regarding the interdependency of homogeneous isodense hematoma and no appearance of recurrence $\left(p=0.038 ; X^{2}-\right.$ independence - test). Maybe, this can be explained by the patients who develop homogeneous isodense hematoma, having an early increase of intracranial pressure because of less pronounced brain atrophy and therefore having an early onset of clinical symptoms with this early stage of cSDH. Accordingly, the patients show a better brain expansion after the evacuation of the hematoma.

The marked increase of cSDH recurrence in the separated hematoma group might be related to incomplete rinsing throughout the emptying or draining process. This way, fresher blood, especially fixed coagula, remains in the subdural space, or in the hematoma cavity. After liquefaction, this may lead to the development of cSDH recurrence. It also explains why the basally located cSDHs relapse more often than those located on the convexity (18).

To the best of our knowledge, no study has been published up to the present day dealing with the $\mathrm{CSDH}$ recurrence in connection with hematoma content. In the present study, we classified the hematoma content according to its properties into four groups: 1. oleaginous 2. bloody, liquid 3 . bloody with coagula 4 . xanthochromic, watery. The most frequently recurring hematoma type was the hematoma with oleaginous contents (35\%), followed by the hematoma with xanthochromic, watery content $(33 \%)$. Chronic SDH with bloody content with or without coagula relapsed much less frequently ( $14 \%$ and $15 \%$, respectively). Unfortunately, we could not detect any significant connection between the contents of the cSDH and the appearance of recurrence.

The location where the hematoma drainage is implanted after emptying the cSDH in the subdural space may play an important role. In the study of Nakaguchi et al., the recurrence rate in conjunction with a frontal position of the drainage was only $5 \%$, while being above $30 \%$ in case of all other positions (19). We found only one study in the literature, which addresses this issue. In our patient population we were not able to detect a significant correlation of the recurrence rate and the position of the drainage, but if no drainage had been implanted, our patients relapsed $\left(p=0.016 ; X^{2}-\right.$ independence - test). Despite our subgroup of patients without a temporary implanted drainage being very small, this finding is in accordance with the results of several authors $(10,13,27,28)$ and may be caused by the postoperative accumulation of air in the subdural space, which prevents the brain from expanding and leads to a more frequent development of $\mathrm{CSDH}$ recurrences $(16,17,20)$. In addition, the early postoperative mobilization with a still implanted subdural drainage might be an important issue in the development and accumulation of subdural air, which promotes the formation of cSDH recurrence $(16,17,20)$.

Concerning the impact of anticoagulants and anti-platelet agents on the development of cSDH recurrences, there have been controversial reports. Forster et al. found a significant correlation of preoperative aspirin intake with recurrence operations (5). However, Lindvall and Koskinen saw no link between the preoperative medication with anticoagulants and the probability of CSDH recurrence development (11). Regarding warfarin application, Gonugunta and Buxton did not find an increase of the cSDH recurrence rate (6). Patients taking anticoagulants or anti-platelet agents are much more frequently represented in the collectives with primary $\mathrm{CSDH}$, which is not the case for the collectives exhibiting $\mathrm{CSDH}$ recurrence $(11,22,29)$. In our study there was no significance regarding the association between the intake of any type of anticoagulants or anti-platelet agents and the appearance of recurrences.

\section{CONCLUSION}

With the data obtained in this prospective study, we were able to point out some significant predictors of recurrence of cSDH. We determined speech disorders, separated presentation of the $\mathrm{CSDH}$ in the preoperative CCTs and no drainage implant to be associated with the development of cSDH recurrence. Homogeneous isodense cSDH in the preoperative CCT was a good prognostic sign regarding the potential recurrence appearance.

Not all gained information of our study about recurrence of cSDH is novel. The association of separated SDH and a higher recurrence rate had also been detected by other authors previously $(18,25)$. However, the dependence of aphasia on the appearance of recurrence has, to our knowledge, not been reported, so far. All patients with aphasia exhibited a left-sided chronic subdural hematoma and most hematomas showed a significant frontobasal expansion. Interestingly, Stanisic et al. (25) and Nakaguchi et al. (18) detected an association between basal chronic subdural hematoma and recurrence. This may partially explain the correlation of speech disorders with high recurrence rates.

Our findings help obtaining a clearer picture of the risk factors and prognostic symptoms associated with cSDH recurrence. Nonetheless, randomized multicentric data with much more study power are mandatory to confirm our data and to gain more possible associations of recurrence like the drainage position and the hematoma content. With our not randomized single center prospective study with its limited number of cases we were not able to detect a significant connection between the occurrence of cSDH recurrence, the drainage position and hematoma content. We are convinced, however, that in the long run our data help optimize the individual treatment of future cSDH patients. 


\section{- REFERENCES}

1. Abouzari M, Rashidi A, Rezaii J, Esfandiari Kh, Asadollahi M, Aleali $\mathrm{H}$, Abdollahzadeh $\mathrm{M}$ : The role of postoperative patient posture in the recurrence of traumatic chronic hematoma after burr-hole surgery. Neurosurgery 61: 794-797, 2007

2. Althoff H, Krenkel W, Schroder J: Raumfordernde Hämatome der hinteren Schädelgrube. Beitr Gerichtl Med 40: 395-401, 1982 (In German)

3. Destandau J, Dartigues JF, Cohadon F: Chronic subdural hematoma in adult. Prognostic factors of surgery. Apropos of 100 cases. Neurosurgery 33(1): 17-22, 1987

4. Ernestus Rl, Beldzinski P, Lanfermann H, Klug N: Chronic subdural hematoma: Surgical treatment and outcome in 104 patients. Surg Neurol 48: 220-225, 1997

5. Forster MT, Mathé AK, Senft C, Scharrer I, Seifert V, Gerlach $R$ : The influence of preoperative anticoagulation on outcome and quality of life after surgical treatment of chronic subdural hematoma. J Clin Neurosci 17(8): 975-979, 2010

6. Gonugunta V, Buxton N: Warfarin and chronic subdural haematomas. Br J Neurosurg 15: 514-517, 2001

7. Ito $\mathrm{H}$, Yamamoto $\mathrm{S}$, Komai $\mathrm{T}$, Mizukoshi $\mathrm{H}$ : Role of local hyperfibrinolysis in the etiology of chronic subdural hematoma. J Neurosurg 45: 26-31, 1976

8. Kang HL, Shin HS, Kim TH, Hwang YS, Park SK: Clinical analysis of recurrent chronic subdural hematoma. J Korean Neurosurg Soc 40: 262-266, 2006

9. Ko BS, Lee Jk, Seo BR, Moon SJ, Kim JH, Kim SH: Clinical analysis of risk factors to recurrent chronic subdural hematoma. J Korean Neurosurg Soc 42:11-15, 2008

10. Lind CR, Lind CJ, Mee EW: Reduction in the number of repeated operations for the treatment of subacute and chronic subdural hematomas by placement of subdural drains. J Neurosurg 99:44-46, 2003

11. Lindvall P, Koskinen LO: Anticoagulants and antiplatelets agents and the risk of development and recurrencs of chronic subdural haematomas. J Clin Neurosci 16(10):1287-1290, 2009

12. Markwalder TM: Chronic subdural hematomas: A review. J Neurosurg 54: 637-645, 1981

13. Markwalder TM, Seiler RW: Chronic subdural hematomas: To drain or not to drain? Neurosurgery 16: 185-188, 1985

14. Matsumoto K, Akagi K, Abekura M, Ryujin H, Ohkawa M, Iwasa N, Akiyama C: Recurrence factors for chronic subdural hematomas after burr-hole craniostomy and closed system drainage. Neurol Res 21: 277-280, 1999

15. Mellergard P, Wisten O: Operations and re-operations for chronic subdural haematomas during a 25-year period in a well defined population. Acta Neurochir 138: 708-713, 1996
16. Merlicco G, Pierangeli E, di Padova PL: Chronic subdural hematomas in adults: Prognostic factors. Analysis of 70 cases. Neurosurg Rev 18: 247-251, 1995

17. Mori K, Maeda M: Surgical treatment of chronic subdural hematoma in 500 consecutive cases: Clinical characteristics, surgical outcome, complications and recurrence rate. Neurol Med Chir (Tokyo) 41: 371-381, 2001

18. Nakaguchi $\mathrm{H}$, Tanishima T, Yoshimasu N: Factors in the natural history of chronic subdural hematomas that influence their postoperative recurrence. J Neurosurg 95: 256-262, 2001

19. Nakaguchi H, Tanishima T, Yoshimasu N: Relationship between drainage catheter location and postoperative recurrence of chronic subdural hematoma after burr-hole irrigation and closed-system drainage. J Neurosurg 93: 791-795, 2000

20. Oishi M, Toyama M, Tamatani S, Kitazawa T, Saito M: Clinical factors of recurrent chronic subdural hematoma. Neurol Med Chir (Tokyo) 41: 382-386, 2001

21. Pencalet P: Clinical forms and prognostic factors of chronic subdural hematoma in adult. Neurochirurgie 47(5): 469-472, 2001 (In French)

22. Reymond MA, Marbet G, Radu EW, Gratzl O: Aspirin as a risk factor for hemorrhage in patients with head injuries. Neurosurg Rev 15: 21-25, 1992

23. Sambasivan M: An overview of chronic subdural hematoma: Experience with 2300 cases. Surg Neurol 47: 418-422, 1997

24. Spallone A, Giuffre R, Gagliardi FM, Vagnozzi R: Chronic subdural hematoma in extremely aged patients. Eur Neurol 29: 18-22, 1989

25. Stanisic M, Lund-Johansen M, Mahesparan R: Treatment of chronic subdural hematoma by burr-hole craniostomy in adults: Influence of some factors on postoperative recurrence. Acta Neurochir (Wien) 147: 1249-1257, 2005

26. Taussky P, Fandino J, Landolt H: Number of burr holes as independent predictor of postoperative recurrence in chronic subdural hematoma. Br J Neurosurg 22(2): 279-282, 2008

27. Tsutsumi K, Maeda K, lijima A, Usui M, Okada Y, Kirino T: The relationship of preoperative magnetic resonance imaging findings and closed system drainage in the recurrence of chronic subdural hematoma. J Neurosurg 87: 870-875, 1997

28. Weigel R, Schmiedek P, Krauss JK: Outcome of contemporary surgery for chronic subdural haematoma: Evidence based review. J Neurol Neurosurg Psychiatry 74: 937-943, 2003

29. Wintzen AR: The clinical course of subdural haematoma. A retrospective study of aetiological, chronological and pathological features in 212 patients and a proposed classification. Brain 103: 855-867, 1980

30. Yamamoto $H$, Hirashima $Y$, Hamada $H$, Hayashi N, Origasa $\mathrm{H}$, Endo S: Independent predictors of recurrence of chronic subdural hematoma: Results of multivariate analysis performed using a logistic regression model. J Neurosurg 98: 1217-1221, 2003 